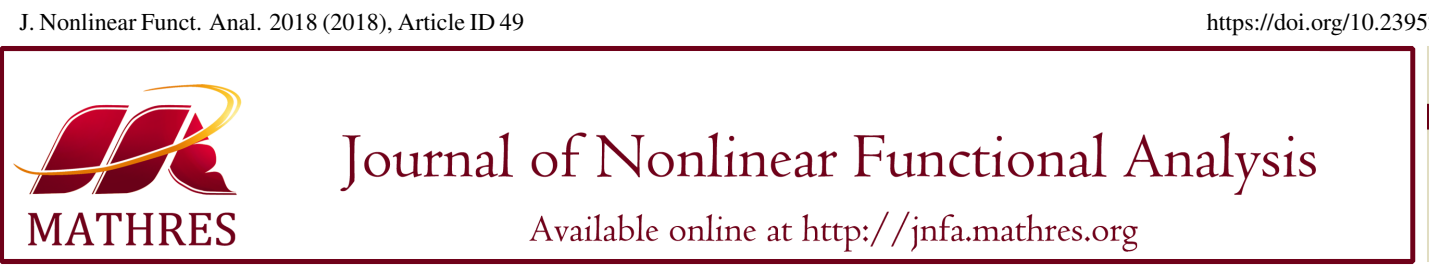

https://doi.org/10.23952/jnfa.2018.49

\title{
A SELF-ADAPTIVE HYBRID STEEPEST DESCENT ALGORITHM FOR SOLVING A CLASS OF VARIATIONAL INEQUALITIES
}

\author{
SONGNIAN HE ${ }^{1,2}$, LILI LIU ${ }^{2}$, XIAOLONG QIN 3 ,* \\ ${ }^{1}$ Tianjin Key Laboratory for Advanced Signal Processing, Civil Aviation University of China, Tianjin 300300, China \\ ${ }^{2}$ College of Science, Civil Aviation University of China, Tianjin 300300, China \\ ${ }^{3}$ Institute of Fundamental and Frontier Sciences, University of Electronic Science and Technology of China, Chengdu, China
}

\begin{abstract}
Let $\mathscr{H}$ be a real Hilbert space. In this paper, we propose a new self-adaptive hybrid steepest descent algorithm for solving a variational inequality problem $\operatorname{VI}(\operatorname{Fix}(T), F)$, were $F: \mathscr{H} \rightarrow \mathscr{H}$ is a boundedly Lipschitz continuous (i.e., Lipschitz continuous on any bounded subset of $\mathscr{H}$ ) and strongly monotone operator and $T: \mathscr{H} \rightarrow \mathscr{H}$ is a nonexpansive mapping with a nonempty fixed point set Fix $(T)$. The strong convergence of our proposed algorithm is proved and the convergence rate estimation is also obtained. The advantage of our algorithm is that it does not require a priori knowledge of the Lipschitz constant of $F$ on any bounded subset of $\mathscr{H}$ and also the strong monotone coefficient.
\end{abstract}

Keywords. Variational inequality; Self-adaptive iterative algorithm; Hybrid steepest descent algorithm; Boundedly Lipschitz continuous; Strongly monotone operator.

AMS Subject Classification: 47J20, 90C25, 90C30, 90C52.

\section{INTRODUCTION}

Let $\mathscr{H}$ be a real Hilbert space with inner product $\langle x, y\rangle$ and induced norm $\|x\|=\sqrt{\langle x, x\rangle}$ for $x, y \in H$. Let $C$ be a nonempty closed and convex subset of $\mathscr{H}$ and let $F: \mathscr{H} \rightarrow \mathscr{H}$ be a nonlinear operator. Recall that the following classical variational inequality problem is to find some $x^{*} \in C$ such that

$$
\left\langle F x^{*}, x-x^{*}\right\rangle \geq 0, \quad \forall x \in C .
$$

In this paper, we use $\mathrm{VI}(C, F)$ to denote the solution set of variational inequality problem (1.1). Variational inequality problem (1.1) was first introduced by Stampacchia [1] in 1964. Since then, it has been extensively studied and applied in a wide variety of problems arising in different fields, for example, engineering sciences, structural analysis, economics, optimization, operations research, see $[2,3,4,5,6$, $7,8,9,10,11]$ and the references therein.

\footnotetext{
* Corresponding author.
}

E-mail addresses: songnianhe@163.com (S. He), 1642692963@qq.com (L. Liu), qxlxajh@ 163.com (X. Qin).

Received October 4, 2018; Accepted December 21, 2018.

(C)2018 Journal of Nonlinear Functional Analysis 
Recently, much attention has been given to develop efficient and implementable numerical methods including projection methods and their variant forms for solving the variational inequality and related optimization problems; see, e.g., $[12,13,14,15,16]$ and the references therein. Since there are no analytic expressions for the metric projection operator in most cases, projection methods and their variant forms are usually inefficient.

The hybrid steepest descent (HSD) method, which was originally proposed by Deutsch and Yamada [17] to avoid the possible projection operators, can be used to solve $\operatorname{VI}(C, F)$ with $C=F i x(T) \neq \emptyset$, where $T$ is a nonexpansive self mapping on $\mathscr{H}$ (see Section 2) and $F i x(T):=\{x \in \mathscr{H} \mid x=T x\}$ is the fixedpoint set of of $T$. With Lipshitz continuous and strongly monotone $F: \mathscr{H} \rightarrow \mathscr{H}$ (see Section 2), the HSD method has been extensively investigated by He and Tian [15], Zhou and Wang [18], Yamada [19], Yang and He [20], Cegielski and Zalas [21], Yamada and Ogura [22], Hirstoaga [23], Takahashi and Yamada [24], and Gibali, Reich and Zalas [25]. With boundedly Lipshitz continuous (i.e., Lipschitz continuous on any bounded subset of $\mathscr{H}$ ) and strongly monotone $F: \mathscr{H} \rightarrow \mathscr{H}$, the HSD method was also studied by $\mathrm{He}$ and $\mathrm{Xu}[26]$ under the assumption that the strong monotone coefficient $\eta$ and Lipschitz constant $L_{B}$ restricted on a bounded subset $B$ of $\mathscr{H}$ are known.

The main purpose of this paper is to propose a new self-adaptive hybrid steepest descent algorithm for solving the variational inequality problem $\mathrm{VI}(\operatorname{Fix}(T), F)$ governed by boundedly Lipshitz continuous and strongly monotone operator $F: \mathscr{H} \rightarrow \mathscr{H}$. The advantage of our algorithm is that it does not require a priori knowledge of the Lipschitz constant of $F$ on any bounded subset of $\mathscr{H}$ and also the strong monotone coefficient. The organization of this paper is as follows. In Section 2, we provide some necessary mathematical preliminaries. In Section 3, the last section, we give the convergence analysis of our self-adaptive hybrid steepest descent algorithm. The convergence rate estimation is also obtained in this section.

\section{PRELIMINARIES}

In this section, we list some concepts and tools that will be used in the proofs of our main results.

Definition 2.1. Let $\mathscr{H}$ be a real Hilbert space.

(i) A mapping $T: \mathscr{H} \rightarrow \mathscr{H}$ is said to be nonexpansive iff

$$
\|T x-T y\| \leq\|x-y\|, \forall x, y \in \mathscr{H}
$$

(ii) A mapping $F: \mathscr{H} \rightarrow \mathscr{H}$ is said to be boundedly Lipschitz continuous iff $F$ is Lipschitz continuous restricted to any bounded subset $B$ of $\mathscr{H}$, i.e., there exists some $L_{B}>0\left(L_{B}\right.$ is relevant with subset $B$ ), such that

$$
\|F x-F y\| \leq L_{B}\|x-y\|, \quad \forall x, y \in B .
$$

Particularly, $F$ is said to be Lipschitz continuous iff there exists a positive constant $L$ such that

$$
\|F x-F y\| \leq L\|x-y\|, \quad \forall x, y \in \mathscr{H} ;
$$

(iii) A mapping $F: \mathscr{H} \rightarrow \mathscr{H}$ is said to be monotone iff

$$
\langle F x-F y, x-y\rangle \geq 0, \forall x, y \in \mathscr{H} ;
$$

(iv) A mapping $F: \mathscr{H} \rightarrow \mathscr{H}$ is said to be strongly monotone iff

$$
\langle F x-F y, x-y\rangle \geq \eta\|x-y\|^{2}, \forall x, y \in \mathscr{H} ;
$$


Remark 2.2. We remark here that the exists of fixed points of the class of nonexpansive mappings was proved by Browder [27]. There is a complementary relationship with the class of nonexpansive mappings and the class of monotone mappings, that is, $T$ is nonexpansive if and only if $I-T$ is monotone.

Lemma 2.3. The following inequality holds in Hilbert spaces:

$$
\|x+y\|^{2} \leq\|x\|^{2}+2\langle y, x+y\rangle, \forall x, y \in \mathscr{H} .
$$

Lemma 2.4. [28] Let $C$ be a nonempty closed convex subset of a real Hilbert space $\mathscr{H}$ and let $T: C \rightarrow C$ be a nonexpansive mapping. Then $I-T$ is demiclosed at 0 in the sense that if $\left\{x_{n}\right\}_{n=1}^{\infty}$ is a sequence in $C$ such that $x_{n} \rightarrow x$ and $\left\|x_{n}-T x_{n}\right\| \rightarrow 0$ as $n \rightarrow \infty$, it follows that $x-T x=0$, i.e., $x \in$ Fix $(T)$. Here $\operatorname{Fix}(T)=\{x \in H \mid T x=x\}$ is the set of fixed points of $T$.

Lemma 2.5. [29] Assume $\left\{a_{n}\right\}_{n=0}^{\infty}$ is a sequence of nonnegative real numbers such that

$$
a_{n+1} \leq\left(1-\gamma_{n}\right) a_{n}+\gamma_{n} \sigma_{n}, n \geq 0,
$$

where $\left\{\gamma_{n}\right\}_{n=0}^{\infty}$ is a sequence in $(0,1)$ and $\left\{\sigma_{n}\right\}_{n=0}^{\infty}$ is a sequence of real numbers such that

(i) $\sum_{n=0}^{\infty} \gamma_{n}=\infty$,

(ii) $\sum_{n=1}^{\infty}\left|\gamma_{n} \sigma_{n}\right|=\infty$, or $\lim \sup _{n \rightarrow \infty} \sigma_{n} \leq 0$.

Then $\lim _{n \rightarrow \infty} a_{n}=0$.

Lemma 2.6. [26] Let $C$ be a nonempty closed convex subset of a real Hilbert space $\mathscr{H}$. If $F: C \rightarrow \mathscr{H}$ is a strongly monotone and boundedly Lipschitz continuous operator, then the variational inequality $\operatorname{VI}(C, F)$ has a unique solution.

In the rest of this paper, we always denote by $\mathscr{H}$ a real Hilbert space and denote by $I$ the identity operator on $\mathscr{H}$. Also, we will use the following notations:

(i) $\rightarrow$ denotes strong convergence.

(ii) $\rightarrow$ denotes weak convergence.

(iii) $\omega_{w}\left(x_{n}\right)=\left\{x \mid \exists\left\{x_{n_{k}}\right\}_{k=1}^{\infty} \subset\left\{x_{n}\right\}_{n=1}^{\infty}\right.$ such that $\left.x_{n_{k}} \rightarrow x\right\}$ denotes the weak $\omega$-limit set of $\left\{x_{n}\right\}_{n=1}^{\infty}$.

\section{MAIN RESUlTS}

In this section, we propose a self-adaptive hybrid steepest descent algorithm for solving $\mathrm{VI}(C, F)$, where $C=F i x(T)$ is the nonempty fixed point set of some nonexpansive mapping $T: \mathscr{H} \rightarrow \mathscr{H}$ and $F: \mathscr{H} \rightarrow \mathscr{H}$ is boundedly Lipschitz continuous and strongly monotone. It is well-known that Fix $(T)$ is a closed convex subset of $\mathscr{H}$ and hence the projection operator $P_{F i x(T)}$ is well defined. Also using Lemma 2.6, we assert that $\mathrm{VI}(C, F)$ has a unique solution.

Algorithm 3.1. (The Self-Adaptive Hybrid Steepest Descent Algorithm)

Step 1: Choose $x_{0}, x_{1} \in \mathscr{H}$ arbitrarily such that $x_{0} \neq x_{1}$, and set $n:=1$.

Calculate

$$
\begin{gathered}
\eta_{0}=\frac{\left\langle F\left(x_{1}\right)-F\left(x_{0}\right), x_{1}-x_{0}\right\rangle}{\left\|x_{1}-x_{0}\right\|^{2}}, \\
L_{0}=\frac{\left\|F\left(x_{1}\right)-F\left(x_{0}\right)\right\|}{\left\|x_{1}-x_{0}\right\|} \text {, and } \mu_{0}=\frac{\eta_{0}}{L_{0}^{2}} .
\end{gathered}
$$


Step 2: For the current $x_{n}$, calculate

$$
\begin{gathered}
\eta_{n}= \begin{cases}\eta_{n-1}, & \text { if } x_{n}=T^{n-1} x_{0} ; \\
\min \left\{\eta_{n-1}, \frac{\left\langle F\left(x_{n}\right)-F\left(T^{n-1} x_{0}\right), x_{n}-T^{n-1} x_{0}\right\rangle}{\left\|x_{n}-T^{n-1} x_{0}\right\|^{2}}\right\}, & \text { if } x_{n} \neq T^{n-1} x_{0},\end{cases} \\
L_{n}= \begin{cases}L_{n-1}, & \text { if } x_{n}=T^{n-1} x_{0} ; \\
\max \left\{L_{n-1}, \frac{\left\|F\left(x_{n}\right)-F\left(T^{n-1} x_{0}\right)\right\|}{\left\|x_{n}-T^{n-1} x_{0}\right\|}\right\}, & \text { if } x_{n} \neq T^{n-1} x_{0},\end{cases}
\end{gathered}
$$

and

$$
\mu_{n}=\frac{\eta_{n}}{L_{n}^{2}}
$$

Step 3: Update the new iterate

$$
x_{n+1}=T\left(I-\lambda_{n} \mu_{n} F\right) x_{n},
$$

where $\lambda_{n} \in(0,1)$. Set $n:=n+1$ and return to Step 2 .

Theorem 3.2. Assume that $F: \mathscr{H} \rightarrow \mathscr{H}$ is boundedly Lipschitz continuous and strongly monotone and the sequence $\left\{\lambda_{n}\right\}_{n=1}^{\infty}$ satisfies the following:

(i) $\lim _{n \rightarrow \infty} \lambda_{n}=0$;

(ii) $\sum_{n=1}^{\infty} \lambda_{n}=\infty$;

(iii) $\sum_{n=1}^{\infty}\left|\lambda_{n+1}-\lambda_{n}\right|<\infty$, or $\lim _{n \rightarrow \infty} \frac{\lambda_{n+1}}{\lambda_{n}}=1$.

Then the sequence $\left\{x_{n}\right\}_{n=0}^{\infty}$ generated by Algorithm 3.1 converges strongly to the unique solution $x^{*}$ of problem 1.1.

Proof. First of all, we prove the boundedness of the sequence $\left\{x_{n}\right\}_{n=0}^{\infty}$. For each $n \geq 1$, put $y_{n+1}=$ $T\left(I-\lambda_{n} \mu_{n} F\right) T^{n-1} x_{0}$. Since $T$ is nonexpansive and using the definitions of $\eta_{n}, L_{n}$ and $\mu_{n}$, we deduce that

$$
\begin{aligned}
\left\|x_{n+1}-y_{n+1}\right\|^{2}= & \left\|T\left(I-\lambda_{n} \mu_{n} F\right) x_{n}-T\left(I-\lambda_{n} \mu_{n} F\right) T^{n-1} x_{0}\right\|^{2} \\
\leq & \left\|\left(x_{n}-T^{n-1} x_{0}\right)-\lambda_{n} \mu_{n}\left(F\left(x_{n}\right)-F\left(T^{n-1} x_{0}\right)\right)\right\|^{2} \\
\leq & \left\|x_{n}-T^{n-1} x_{0}\right\|^{2}-2 \lambda_{n} \mu_{n}\left\langle F\left(x_{n}\right)-F\left(T^{n-1} x_{0}\right), x_{n}-T^{n-1} x_{0}\right\rangle \\
& +\lambda_{n}^{2} \mu_{n}^{2}\left\|F\left(x_{n}\right)-F\left(T^{n-1} x_{0}\right)\right\|^{2} \\
\leq & \left\|x_{n}-T^{n-1} x_{0}\right\|^{2}-2 \lambda_{n} \mu_{n} \eta_{n}\left\|x_{n}-T^{n-1} x_{0}\right\|^{2}+\lambda_{n}^{2} \mu_{n}^{2} L_{n}^{2}\left\|x_{n}-T^{n-1} x_{0}\right\|^{2} \\
\leq & \left(1-2 \lambda_{n} \frac{\eta_{n}^{2}}{L_{n}^{2}}+\lambda_{n}^{2} \frac{\eta_{n}^{2}}{L_{n}^{2}}\right)\left\|x_{n}-T^{n-1} x_{0}\right\|^{2} \\
= & {\left[1-\lambda_{n} \frac{\eta_{n}^{2}}{L_{n}^{2}}\left(2-\lambda_{n}\right)\right]\left\|x_{n}-T^{n-1} x_{0}\right\|^{2} } \\
\leq & {\left[1-\frac{\lambda_{n} \eta_{n}^{2}}{2 L_{n}^{2}}\left(2-\lambda_{n}\right)\right]^{2}\left\|x_{n}-T^{n-1} x_{0}\right\|^{2} . }
\end{aligned}
$$

Consequently, we get

$$
\left\|x_{n+1}-y_{n+1}\right\| \leq\left[1-\frac{\lambda_{n} \eta_{n}^{2}}{2 L_{n}^{2}}\left(2-\lambda_{n}\right)\right]\left\|x_{n}-T^{n-1} x_{0}\right\| .
$$


Obviously, we also have

$$
\begin{aligned}
\left\|y_{n+1}-T^{n} x_{0}\right\| & =\left\|T\left(I-\lambda_{n} \mu_{n} F\right) T^{n-1} x_{0}-T^{n} x_{0}\right\| \\
& \leq \lambda_{n} \mu_{n}\left\|F\left(T^{n-1} x_{0}\right)\right\| .
\end{aligned}
$$

Combining (3.2) and (3.3) yields

$$
\begin{aligned}
\left\|x_{n+1}-T^{n} x_{0}\right\| & \leq\left\|x_{n+1}-y_{n+1}\right\|+\left\|y_{n+1}-T^{n} x_{0}\right\| \\
& \leq\left[1-\frac{\lambda_{n} \eta_{n}^{2}}{2 L_{n}^{2}}\left(2-\lambda_{n}\right)\right]\left\|x_{n}-T^{n-1} x_{0}\right\|+\lambda_{n} \mu_{n}\left\|F\left(T^{n-1} x_{0}\right)\right\| .
\end{aligned}
$$

Taking into account that

$$
\left\|T^{n} x_{0}-x^{*}\right\| \leq\left\|T^{n-1} x_{0}-x^{*}\right\| \leq\left\|x_{0}-x^{*}\right\|,
$$

we find that $\left\{T^{n} x_{0}\right\}_{n=1}^{\infty}$ is bounded. Thus we assert that $\left\{\left\|F\left(T^{n-1} x_{0}\right)\right\|\right\}_{n=1}^{\infty}$ is also bounded since $F$ is boundedly Lipschitz continuous, i.e., $M=\sup _{n \geq 1}\left\|F\left(T^{n-1} x_{0}\right)\right\|<+\infty$. Hence, for all $n \geq 1$, we find from (3.4) that

$$
\begin{aligned}
\left\|x_{n+1}-T^{n} x_{0}\right\| & \leq\left[1-\frac{\lambda_{n} \eta_{n}^{2}}{2 L_{n}^{2}}\left(2-\lambda_{n}\right)\right]\left\|x_{n}-T^{n-1} x_{0}\right\|+\lambda_{n} \mu_{n} M \\
& \leq \max \left\{\left\|x_{n}-T^{n-1} x_{0}\right\|, \frac{2 M}{\eta_{n}}\right\} \\
& \leq \max \left\{\left\|x_{n}-T^{n-1} x_{0}\right\|, \frac{2 M}{\eta}\right\} \\
& \leq \max \left\{\left\|x_{1}-x_{0}\right\|, \frac{2 M}{\eta}\right\} .
\end{aligned}
$$

This means that $\left\{x_{n}\right\}_{n=0}^{\infty}$ is bounded, so is $\left\{T x_{n}\right\}_{n=0}^{\infty}$.

Next, we turn to proving $\left\|x_{n}-T x_{n}\right\| \rightarrow 0(n \rightarrow \infty)$ and $\omega\left(x_{n}\right) \subset$ Fix $(T)$. Using (3.1), we deduce

$$
\begin{aligned}
\left\|x_{n+1}-x_{n}\right\| & =\left\|T\left(I-\lambda_{n} \mu_{n} F\right) x_{n}-T\left(I-\lambda_{n-1} \mu_{n-1} F\right) x_{n-1}\right\| \\
& \leq\left\|\left(x_{n}-x_{n-1}\right)-\lambda_{n} \mu_{n}\left(F\left(x_{n}\right)-F\left(x_{n-1}\right)\right)+\left(\lambda_{n-1} \mu_{n-1}-\lambda_{n} \mu_{n}\right) F\left(x_{n-1}\right)\right\| \\
& \leq\left\|\left(x_{n}-x_{n-1}\right)-\lambda_{n} \mu_{n}\left(F\left(x_{n}\right)-F\left(x_{n-1}\right)\right)\right\|+\left|\lambda_{n-1} \mu_{n-1}-\lambda_{n} \mu_{n}\right|\left\|F\left(x_{n-1}\right)\right\| .
\end{aligned}
$$

Put $B=\overline{\mathrm{co}}\left\{x^{*}, x_{0}, x_{1}, \cdots, x_{n}, \cdots\right\}$, the closed convex hull containing $x^{*}$ and $\left\{x_{n}\right\}_{n=0}^{\infty}$. since $\left\{x_{n}\right\}_{n=0}^{\infty}$ is bounded, we see that $B$ is a bounded closed convex subset of $\mathscr{H}$. Then $F$ is Lipschitz continuous on $B$, i.e., there exists some $L_{B}>0$ such that

$$
\|F(x)-F(y)\| \leq L_{B}\|x-y\|, \quad \forall x, y \in B .
$$

Particularly, we have

$$
\left\|F\left(x_{n}\right)-F\left(x_{n-1}\right)\right\| \leq L_{B}\left\|x_{n}-x_{n-1}\right\| .
$$

This together with

$$
\left\langle F\left(x_{n}\right)-F\left(x_{n-1}\right), x_{n}-x_{n-1}\right\rangle \geq \eta\left\|x_{n}-x_{n-1}\right\|^{2}
$$


leads to

$$
\begin{aligned}
& \left\|\left(x_{n}-x_{n-1}\right)-\lambda_{n} \mu_{n}\left(F\left(x_{n}\right)-F\left(x_{n-1}\right)\right)\right\|^{2} \\
& =\left\|x_{n}-x_{n-1}\right\|^{2}-2 \lambda_{n} \mu_{n}\left\langle F\left(x_{n}\right)-F\left(x_{n-1}\right), x_{n}-x_{n-1}\right\rangle+\lambda_{n}^{2} \mu_{n}^{2}\left\|F\left(x_{n}\right)-F\left(x_{n-1}\right)\right\|^{2} \\
& \leq\left(1-2 \lambda_{n} \mu_{n} \eta+\lambda_{n}^{2} \mu_{n}^{2} L_{B}^{2}\right)\left\|x_{n}-x_{n-1}\right\|^{2} \\
& =\left[1-\lambda_{n} \mu_{n}\left(2 \eta-\lambda_{n} \mu_{n} L_{B}^{2}\right)\right]\left\|x_{n}-x_{n-1}\right\|^{2} \\
& \leq\left[1-\lambda_{n} \mu_{n} \gamma_{n}\right]^{2}\left\|x_{n}-x_{n-1}\right\|^{2},
\end{aligned}
$$

where $\gamma_{n}=\eta-\frac{1}{2} \lambda_{n} \mu_{n} L_{B}^{2}$. Combining (3.5) and (3.6), we have

$$
\left\|x_{n+1}-x_{n}\right\| \leq\left[1-\lambda_{n} \mu_{n} \gamma_{n}\right]\left\|x_{n}-x_{n-1}\right\|+\left|\lambda_{n-1} \mu_{n-1}-\lambda_{n} \mu_{n}\right| M,
$$

where $M=\sup \left\{\left\|F\left(x_{n-1}\right)\right\|\right\}_{n=1}^{\infty}<+\infty$. Notice that $\frac{\eta}{L_{B}^{2}} \leq \mu_{n} \leq \frac{1}{\eta}, \lim _{n \rightarrow \infty} \gamma_{n}=\eta$. Using conditions (i)-(iii), it is easy to verify that

$$
\lim _{n \rightarrow \infty} \lambda_{n} \mu_{n} \gamma_{n}=0, \sum_{n=1}^{\infty} \lambda_{n} \mu_{n} \gamma_{n}=\infty
$$

and

$$
\sum_{n=1}^{\infty}\left|\lambda_{n-1} \mu_{n-1}-\lambda_{n} \mu_{n}\right|<\infty, \text { or } \lim _{n \rightarrow \infty} \frac{\left|\lambda_{n-1} \mu_{n-1}-\lambda_{n} \mu_{n}\right|}{\lambda_{n} \mu_{n} \gamma_{n}}=0 .
$$

Hence, using Lemma 2.5, we assert that $\left\|x_{n+1}-x_{n}\right\| \rightarrow 0(n \rightarrow \infty)$. Furthermore, this together with (3.1) leads to

$$
\begin{aligned}
\left\|x_{n}-T x_{n}\right\| & \leq\left\|x_{n}-x_{n+1}\right\|+\left\|x_{n+1}-T x_{n}\right\| \\
& \leq\left\|x_{n}-x_{n+1}\right\|+\lambda_{n} \mu_{n}\left\|F x_{n}\right\| \rightarrow 0(n \rightarrow \infty) .
\end{aligned}
$$

Consequently, it follows from Lemma 2.4 that $\omega\left(x_{n}\right) \subset F i x(T)$.

Finally, we prove $x_{n} \rightarrow x^{*}(n \rightarrow \infty)$. Using (2.6) and (3.1), we have

$$
\begin{aligned}
\left\|x_{n+1}-x^{*}\right\|^{2} & =\left\|T\left(I-\lambda_{n} \mu_{n} F\right) x_{n}-T x^{*}\right\|^{2} \\
& =\left\|\left(I-\lambda_{n} \mu_{n} F\right) x_{n}-\left(I-\lambda_{n} \mu_{n} F\right) x^{*}-\lambda_{n} \mu_{n} F x^{*}\right\|^{2} \\
& \leq\left\|\left(I-\lambda_{n} \mu_{n} F\right) x_{n}-\left(I-\lambda_{n} \mu_{n} F\right) x^{*}\right\|^{2}-2 \lambda_{n} \mu_{n}\left\langle F x^{*}, x_{n}-x^{*}-\lambda_{n} \mu_{n} F x_{n}\right\rangle .
\end{aligned}
$$

Similar to (3.6), we get

$$
\left\|\left(I-\lambda_{n} \mu_{n} F\right) x_{n}-\left(I-\lambda_{n} \mu_{n} F\right) x^{*}\right\| \leq\left(1-\lambda_{n} \mu_{n} \gamma_{n}\right)\left\|x_{n}-x^{*}\right\| .
$$

Combining (3.9) and (3.10) yields

$$
\begin{aligned}
\left\|x_{n+1}-x^{*}\right\|^{2} & \leq\left(1-\lambda_{n} \mu_{n} \gamma_{n}\right)\left\|x_{n}-x^{*}\right\|^{2}-2 \lambda_{n} \mu_{n}\left\langle F x^{*}, x_{n}-x^{*}\right\rangle \\
& +2 \lambda_{n}^{2} \mu_{n}^{2}\left\|F x^{*}\right\|\left\|F x_{n}\right\| \\
& \leq\left(1-\lambda_{n} \mu_{n} \gamma_{n}\right)\left\|x_{n}-x^{*}\right\|^{2}+\lambda_{n} \mu_{n} \gamma_{n} \delta_{n},
\end{aligned}
$$

where

$$
\delta_{n}=\frac{2}{\gamma_{n}}\left\langle F x^{*}, x^{*}-x_{n}\right\rangle+\frac{2 \lambda_{n} \mu_{n}}{\gamma_{n}}\left\|F x^{*}\right\|\left\|F x_{n}\right\| .
$$

From (3.8) and (3.11), in order to complete the proof by using Lemma 2.5, in suffices to verify that $\lim _{n \rightarrow \infty} \sup \delta_{n} \leq 0$. Indeed, we take a subsequence $\left\{x_{n_{k}}\right\}_{k=1}^{\infty} \subset\left\{x_{n}\right\}_{n=1}^{\infty}$ such that

$$
\lim _{n \rightarrow \infty} \sup \frac{2}{\gamma_{n}}\left\langle F x^{*}, x^{*}-x_{n}\right\rangle=\lim _{k \rightarrow \infty} \frac{2}{\gamma_{n_{k}}}\left\langle F x^{*}, x^{*}-x_{n_{k}}\right\rangle
$$


and $x_{n_{k}} \rightarrow \hat{x}$. Noting the fact that $\hat{x} \in \omega\left(x_{n}\right) \subset \operatorname{Fix}(T)$ and $x^{*}$ is the unique solution of $\operatorname{VI}(\operatorname{Fix}(T), F)$, we obtain

$$
\lim _{n \rightarrow \infty} \sup \frac{2}{\gamma_{n}}\left\langle F x^{*}, x^{*}-x_{n}\right\rangle=\lim _{k \rightarrow \infty} \frac{2}{\gamma_{n_{k}}}\left\langle F x^{*}, x^{*}-x_{n_{k}}\right\rangle=\frac{2}{\eta}\left\langle F x^{*}, x^{*}-\hat{x}\right\rangle \leq 0,
$$

and consequently $\lim _{n \rightarrow \infty} \sup \delta_{n} \leq 0$. The proof is complete.

We now focus on the estimation of the convergence rate of Algorithm 3.1 in the non asymptotic sense. Our proof is based on the fundamental fact: a point $z \in C$ is a solution of $\operatorname{VI}(C, F)$ if and only if $\langle F x, x-z\rangle \geq 0$ holds for all $x \in C \bigcap S(z, 1)$, where $S(z, 1)$ is the closed sphere with center $z$ and radius one. (see [10] and [13] for details).

First, we give a fundamental inequality below.

Theorem 3.3. Let $\left\{x_{n}\right\}_{n=1}^{\infty}$ be the sequence generated by Algorithm 3.1. Assume that all conditions in Theorem 3.2 are satisfied, and the condition $\sum_{n=0}^{\infty} \lambda_{n}^{2}<\infty$ is also satisfied. Then, for any integer $n \geq 1$, we have a point $z_{n} \in \mathscr{H}$, such that the sequence $\left\{z_{n}\right\}_{n=1}^{\infty}$ converges strongly to the unique solution $x^{*}$ of $\operatorname{VI}(\operatorname{Fix}(T), F)$ and

$$
\left\langle F x, z_{n}-x\right\rangle \leq \frac{\left\|x_{0}-x\right\|^{2}+\Psi}{\Upsilon_{n}}, \quad \forall x \in F i x(T)
$$

where

$$
\Psi=\sum_{k=0}^{\infty} \lambda_{k}^{2} \mu_{k}^{2}\left\|F x_{k}\right\|^{2}, z_{n}=\frac{\sum_{k=0}^{n} 2 \lambda_{k} \mu_{k} x_{k}}{\Upsilon_{n}} \text { and } \Upsilon_{n}=\sum_{k=0}^{n} 2 \lambda_{k} \mu_{k}
$$

Proof. Since $T$ is nonexpansive, for each $k \geq 0$ and any $x \in$ Fix $(T)$, we find from (3.1) that

$$
\begin{aligned}
\left\|x_{k+1}-x\right\|^{2} & =\left\|T\left(x_{k}-\lambda_{k} \mu_{k} F x_{k}\right)-T x\right\|^{2} \\
& \leq\left\|x_{k}-x-\lambda_{k} \mu_{k} F x_{k}\right\|^{2} \\
& \leq\left\|x_{k}-x\right\|^{2}-2 \lambda_{k} \mu_{k}\left\langle F x_{k}, x_{k}-x\right\rangle+\lambda_{k}^{2} \mu_{k}^{2}\left\|F x_{k}\right\|^{2} .
\end{aligned}
$$

(3.14) together with the monotonicity of $F$ leads to

$$
2 \lambda_{k} \mu_{k}\left\langle F x, x_{k}-x\right\rangle \leq\left\|x_{k}-x\right\|^{2}-\left\|x_{k+1}-x\right\|^{2}+\lambda_{k}^{2} \mu_{k}^{2}\left\|F x_{k}\right\|^{2} .
$$

Taking into account the fact that $\left\{\left\|F x_{n}\right\|\right\}_{n=0}^{\infty}$ is bounded and $\frac{\eta}{L_{B}^{2}} \leq \mu_{n} \leq \frac{1}{\eta}$ for all $n \geq 0$, where

$$
B=\overline{\mathrm{co}}\left\{x^{*}, x_{0}, x_{1}, \cdots, x_{n}, \cdots\right\}
$$

and $L_{B}$ is the Lipschitz constant of $F$ restricted to $B$, we conclude from condition $\sum_{k=0}^{\infty} \lambda_{k}^{2}<\infty$ that $\Psi=\sum_{k=0}^{\infty} \lambda_{k}^{2} \mu_{k}^{2}\left\|F x_{k}\right\|^{2}<\infty$. Summing inequality (3.15) over $k=0, \ldots, n$, we get

$$
\left\langle F x, \sum_{k=0}^{n} 2 \lambda_{k} \mu_{k} x_{k}-\sum_{k=0}^{n} 2 \lambda_{k} \mu_{k} x\right\rangle \leq\left\|x_{0}-x\right\|^{2}+\Psi, \quad \forall x \in F i x(T) .
$$

Thus (3.12) follows from (3.16). By Theorem 3.2, $\left\{x_{n}\right\}_{n=0}^{\infty}$ converges strongly to the unique solution $x^{*}$ of $\operatorname{VI}(F i x(T), F)$. Since $z_{n}$ is a convex combination of $x_{0}, x_{1}, \ldots$, and $x_{n}$, it is easy to see that $\left\{z_{n}\right\}_{n=1}^{\infty}$ also converges strongly to $x^{*}$.

Now, we are in a position to present the convergence rate of Algorithm 3.1. 
Corollary 3.4. Assume that all conditions in Theorem 3.3 are satisfied. In the ergodic sense, Algorithm 3.1 has the $O\left(\frac{1}{n^{1-\alpha}}\right)$ convergence rate if $\left\{\lambda_{n}\right\}_{n=1}^{\infty}=\left\{\frac{1}{n^{\alpha}}\right\}_{n=1}^{\infty}$ with $\frac{1}{2}<\alpha<1$, and has the $O\left(\frac{1}{\ln n}\right)$ convergence rate if $\left\{\lambda_{n}\right\}_{n=1}^{\infty}=\left\{\frac{1}{n}\right\}_{n=1}^{\infty}$.

Proof. Obviously, $\sum_{n=1}^{\infty} \lambda_{n}=\infty$ and $\sum_{n=1}^{\infty} \lambda_{n}^{2}<\infty$ are satisfied if $\left\{\lambda_{n}\right\}_{n=1}^{\infty}=\left\{\frac{1}{n^{\alpha}}\right\}_{n=1}^{\infty}$ with $\frac{1}{2}<\alpha<1$. For any integer $k \geq 1$, it is easy to verify that

$$
\frac{1}{1-\alpha}\left\{(k+1)^{1-\alpha}-k^{1-\alpha}\right\} \leq \frac{1}{k^{\alpha}} .
$$

Consequently, for all $n \geq 1$, we have

$$
\frac{1}{1-\alpha}\left\{(n+1)^{1-\alpha}-1\right\} \leq \sum_{k=1}^{n} \frac{1}{k^{\alpha}} .
$$

Without loss of generality, taking $\lambda_{0}=\frac{1}{1-\alpha}$ and noting $\frac{\eta}{L_{B}^{2}} \leq \mu_{n} \leq \frac{1}{\eta}$ again, it concludes from (3.13) and (3.17) that

$$
\Upsilon_{n} \geq \frac{2 \eta}{L_{B}^{2}(1-\alpha)}(n+1)^{1-\alpha} \geq \frac{2 \eta}{L_{B}^{2}(1-\alpha)} n^{1-\alpha} .
$$

This means that Algorithm 3.1 has the $O\left(\frac{1}{n^{1-\alpha}}\right)$ convergence rate. In fact, for any bounded subset $D \subset$ Fix $(T)$, put $\gamma=\sup \left\{\left\|x_{0}-x\right\|^{2} \mid x \in D\right\}$. Using (3.12) and (3.18), we have

$$
\left\langle F x, z_{n}-x\right\rangle \leq \frac{(\gamma+\Psi)(1-\alpha) L_{B}^{2}}{2 \eta n^{1-\alpha}}, \forall x \in D .
$$

The conclusion can be proved similarly for the case that $\left\{\lambda_{n}\right\}_{n=1}^{\infty}=\left\{\frac{1}{n}\right\}_{n=1}^{\infty}$.

\section{Acknowledgements}

The authors are grateful to the two anonymous referees for their useful suggestions. The first author was supported by Open Fund of Tianjin Key Lab for Advanced Signal Processing (No. 2017ASP-TJ03) and the third author was supported by the National Natural Science Foundation of China under Grant No.11401152.

\section{REFERENCES}

[1] G. Stampacchia, Formes bilineaires coercivites sur les ensembles convexes, C. R. Acad. Sci. 258 (1964), $4413-4416$.

[2] Z. Cai, W. Zhu, Multi-label feature selection via feature manifold learning and sparsity regularization, Int. J. Mach. Learn. Cybernet. 9 (2018), 1321-1334.

[3] S. Wang, W. Zhu, Sparse graph embedding unsupervised feature selection, IEEE Trans. Sys. Man Cybernet. 48 (2018), 329-341.

[4] H. Yang, G. Bell, Traffic restraint, road pricing and network equilibrium, Trans. Res. B. 31 (1997), 303-314.

[5] S.Y. Cho, W. Li, S.M. Kang, Convergence analysis of an iterative algorithm for monotone operators, J. Inequal. Appl. 2013 (2013), Article ID 199.

[6] C. Baiocchi, A. Capelo, Variational and Quasi Variational Inequalities, John Wiley and Sons, New York, 1984.

[7] R.W. Cottle, F. Giannessi, J.L. Lions, Variational Inequalities and Complementarity Problems, Theory and Applications, John Wiley and Sons, New York, 1980.

[8] S.Y. Cho, S.M. Kang, Approximation of fixed points of pseudocontraction semigroups based on a viscosity iterative process, Appl. Math. Lett. 24 (2011), 224-228.

[9] S.Y. Cho, Generalized mixed equilibrium and fixed point problems in a Banach space, J. Nonlinear Sci. Appl. 9 (2016), 1083-1092. 
[10] F. Facchinei, J.S. Pang, Finite-dimensional variational inequalities and complementarity problem, Vol. I and II. Springer Series in Operations Research, Springer-Verlag, New York, 2003.

[11] P.T. Harker, J.S. Pang, Finite-dimensional variational inequality and nonlinear omplementarity problems: a survey of theory, algorithms and applications, Math. Program. 48 (1990), 161-220.

[12] X. Zhao, K.F. Ng, C. Li, J.C. Yao, Linear regularity and linear convergence of projection-based methods for solving convex feasibility problems, Appl. Math. Optim. 78 (2018), 613-641.

[13] X. Cai, G. Gu, B. He, On the $O\left(\frac{1}{t}\right)$ convergence rate of the projection and contraction methods for varitional inequalities with Lipschitz continuous monotone operators, Comput. Optim. Appl. 57 (2014), 339-363.

[14] S.Y. Cho, W. Li, S.M. Kang, Convergence analysis of an iterative algorithm for monotone operators, J. Inequal. Appl. 2013 (2013), Article ID 199.

[15] S. He, H. Tian, Selective projection methods for solving a class of variational inequalities, Numer. Algorithms (2018). https://doi.org/10.1007/s11075-018-0499-x.

[16] S.Y. Cho, S.M. Kang, Approximation of common solutions of variational inequalities via strict pseudocontractions, Acta Math. Sci. 32 (2012), 1607-1618.

[17] F. Deutsch, I. Yamada, Minimizing certain convex functions over the intersection of the fixed point sets of nonexpansive mappings, Numer. Funct. Anal. Optim. 19 (1998), 33-56.

[18] H. Zhou, P. Wang, A simpler explicit iterative algorithm for a class of variational inequalities in Hilbert spaces, J. Optim. Theory Appl. 161 (2014), 716-727.

[19] I. Yamada, The hybrid steepest-descent method for variational inequality problems over the intersection of the fixed point sets of nonexpansive mappings, in: D. Butnariu, Y. Censor, S. Reich (Eds.), Inherently Parallel Algorithms in Feasibility and Optimization and Their Applications, North-Holland, Amsterdam, pp. 473-504, 2001.

[20] C. Yang, S. He, Convergence of explicit iterative algorithms for solving a class of variational inequalities, J. WSEAS Trans. 13 (2014), 830-839.

[21] A. Cegielski, R. Zalas, Methods for variational inequality problem over the intersection of fixed point sets of quasinonexpansive operators, Numer. Funct. Anal. Optim. 34 (2013), 255-283.

[22] I. Yamada, N. Ogura, Hybrid steepest descent method for variational inequality problem over the fixed point set of certain quasi-nonexpansive mappings, Numer. Funct. Anal. Optim. 25 (2004), 619-655.

[23] S.A. Hirstoaga, Iterative selection methods for common fixed point problems, J. Math Anal Appl. 324 (2006), 1020-1035.

[24] N. Takahashi, I. Yamada, Parallel algorithms for variational inequalities over the Cartesian product of the intersections of the fixed point sets of nonexpansive mappings, J. Approx. Theory. 153 (2008), 139-160.

[25] A. Gibali, S. Reich, R. Zalas, Outer approximation methods for solving variational inequalities in Hilbert space, Optimization 66 (2017), 417-437.

[26] S. He, H.K. Xu, Variational inequalities governed by boundedly Lipschitzian and strongly monotone operators, Fixed Point Theory 10 (2009), 245-258.

[27] F.E. Browder, Nonexpansive nonlinear operators in a Banach space, Proc. Natl. Acad. Sci. USA 54 (1965), $1041-1044$.

[28] Z. Opial, Weak convergence of the sequence of successive approximations of nonexpansive mappings, Bull. Amer. Math. Soc. 73 (1967), 595-597.

[29] Z.Q. Xue, H.Y. Zhou, Y.J. Cho, Iterative solutions of nonlinear equations for m-accretive operators in Banach spaces, J. Nonlinear Convex Anal. 1 (2000), 313-320. 